\title{
О ЗАПОЛНЕНИИ РАСТУЩИХ ДЕРЕВЬЕВ ИСТОЧНИКОМ, НАХОДЯЩЕМСЯ В КОРНЕ
}

\author{
В. В. Орлов \\ Общество с ограниченной ответственностью «Гарантстрой»
}

Поступила в редакцию 04.07.2019 г.

\begin{abstract}
Аннотация. В работе рассмотрена оптимизационная задача о заполнении растущих деревьев источником, находящемся в корне. Построены алгоритмы работы этого источника по заполнению графа минимизирующие время заполнения корневого дерева. Полностью рассмотрен случай, когда количество дуг, исходящих из корня, не превышает 3-х, а мощность источника не превосходит 2. Затем рассмотрены некоторые более общие случаи, когда мощность источника и количество корневых поддеревьев произвольны. Сформулированы и доказаны теоремы о существовании оптимальных параллельных стратегий заполнения дерева для некоторых общих случаев и описаны сами оптимальные стратегии. Предложено улучшение построенного алгоритма заполнения дерева за счёт уменьшения количества переключений мощности источника между корневыми поддеревьями. Для всех результатов приведены иллюстрирующие примеры.

Ключевые слова: методы оптимизации, эффективная заправка, планирование производства, алгоритмы, корневое поддерево, заполнение вершин ориентированного графа.
\end{abstract}

\section{ВВЕДЕНИЕ}

В последнее время наметился переход от собственно теории графов к изучению процессов на графах, когда объектом изучения является не сам граф и его свойства, а процесс рассматриваемый на нем, т. е. сам граф выступает «основой», на которой развивается процесс. Началом такого подхода следует считать работы Л. Форда и Д. Фалкерсона по теории потоков в сетях ([1]). Сегодняшний этап развития такого подхода связан с рассмотрением ресурсных сетей (О. П. Кузнецов, Л. Ю. Жилякова [2-6]) и графов с ограничениями на достижимость (Ерусалимский Я. М., Скороходов В. А. [7-11]). Обзор последних результатов по динамическим графовым моделям и процессам сделан в [12].

В настоящей работе мы продолжим рассмотрение задач о заполнении вершин графа динамическим потоком (начало см. [13]). В отличие от классической оптимизационной задачи о максимальном потоке Форда - Фалкерсона, наша цель состоит не в увеличении потока, протекающего через сеть, а в мини-

(C) Орлов В. В., 2019 мизации времени заполнения вершин и дуг растущего из корня дерева потоком, создаваемым в нём источником, находящемся в корне.

В такой постановке задача может рассматриваться как оптимизационная модель процессов орошения, доставки товаров по сети, использования производственных мощностей.

В работе [13] рассмотрена задача о заполнении вершин ориентированного корневого дерева источником единичной мощности. В работе [14] нами была фактически рассмотрена задача о заполнении такого дерева для случая, когда дерево имеет три корневых поддерева, а подключаемый источник имеет мощность равную 2. Настоящая работа посвящена общим случаям, когда мощность источника $m \geq 2$, а количество корневых поддеревьев может быть любым.

\section{МАТЕРИАЛЫ И МЕТОДЫ ИССЛЕДОВАНИЯ}

Объектом исследования является корневое ориентированное дерево и процесс его заполнения. 


\section{В. В. Орлов}

Определение 1. Корневое ориентированное дерево - ацикличный орграб (ориентированный граф, не содержащий изиклов), в котором только одна вершина имеет нулевую степень захода (в неё не ведут дуги), а все остальные вершины имеют степень захода равную единице (в них ведёт ровно по одной дуге). Вершина с нулевой степенью захода называется корнем дерева, вершины с нулевой степенью исхода (из которых не исходит ни одна дуга) называются концевыми вершинами или листьями.

Определение 2. Рассмотрим корневое ориентированное дерево и произвольную дугу $и$, выходяшую из корня. Вершина у, в которой заканчивается эта дуга, порождает максимальный по вложению корневой подграф $T_{y} c$ корнем в этой вершине. Корневым поддеревом, порожденным дугой и будем называть подграб $T_{\{u\}}=T_{y} \cup\{u\}$.

\section{Пример 1.}

Корневое ордерево, изображённое на рис. 1, состоит из корня и четырёх корневых поддеревьев $T_{\left\{y_{1}\right\}} \cup\left\{u_{1}\right\} ; \quad T_{\left\{y_{2}\right\}} \cup\left\{u_{2}\right\} ; \quad T_{\left\{y_{3}\right\}} \cup\left\{u_{3}\right\} ;$ $T_{\left\{y_{4}\right\}} \cup\left\{u_{4}\right\}$ и $\left(T_{\left\{y_{i}\right\}} \cup\left\{u_{i}\right\}\right) \cap\left(T_{\left\{y_{j}\right\}} \cup\left\{u_{j}\right\}\right)=\left\{x_{0}\right\}$, $i \neq j$.

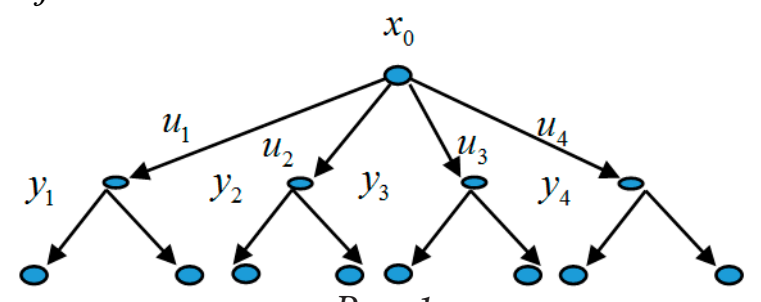

Puc. 1

Далее мы будем рассматривать только корневые поддеревья, а для удобства писать просто «поддеревья» и обозначать их $T_{i}$, где $i$ - порядковый номер поддерева.

Будем считать, что все вершины корневого дерева, кроме корня, имеют положительную целочисленную ёмкость. Ёмкость корневой вершины будем считать равной 0, то есть корневая вершина будет являться транзитёром (см. [13]) на протяжении всего процесса заполнения графа. Ёмкость каждой из дуг графа считается равной единице. Пропускная способность каждой из дуг равна единице. Будем считать, что к корневой вершине подключен источник, мощность которого в каждый момент времени целочислена и не больше чем. Будем считать также, что заполняющий поток в каждый момент времени целочисленен. Рассмотрим задачу о заполнении корневого дерева таким источником. То есть в единицу времени корневая вершина может выдавать две или одну единицу вещества в единицу времени. Определение потока и подробное описание процесса заполнения см. в [13].

Определение 3. Корневое поддерево называется незаполненным в момент времени $t$ если хотя бы одна из его вериин в этот момент времени является потребителем (cM [13]).

Определение 4. Ёмкостью корневого поддерева назовём число с $\left(T_{i}\right)$ равное сумме ёмкостей вериин и дуг, входящих в это поддерево.

\section{РЕЗУЛЬТАТЫ ИССЛЕДОВАНИЙ И ИХ ОБСУЖДЕНИЯ}

\section{$\mathbf{1}^{0}$. Задача о заполнении растущего кор-} невого дерева с вершинами положительной емкости одним источником мощности 2.

В силу сказанного выше в процессе заполнения источник работает не более чем на два поддерева одновременно, при работе источника только на одно поддерево его мощность снижается до единичной, а при работе на два поддерева, его мощность равна двум и на каждое из этих поддеревьев он (источник) работает с единичной мощностью.

Любые два незаполненных поддерева графа могут заполняться одновременно. Процесс заполнения, при котором мощность источника используется полностью на протяжении всего времени заправки, будем называть параллельным. Ясно, что при параллельном процессе заполнения в каждый момент времени осуществляется заполнение каких-то двух поддеревьев.

Если параллельный процесс существует, то он будет оптимальным по времени заполнения рассматриваемого корневого дерева, а сам процесс параллельного заполнения будем называть параллельной стратегией. Ниже будет показано, что не на всяком корневом дереве такой процесс существует. Для таких деревьев в работе также будет предложена оп- 


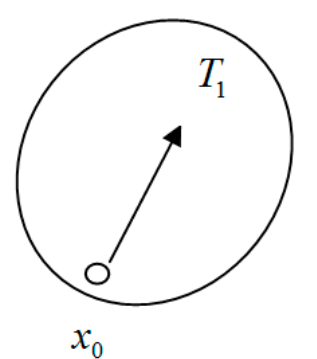

$x_{0}$

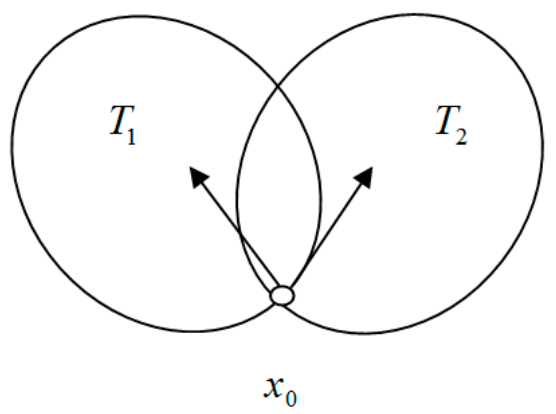

Puc. 2

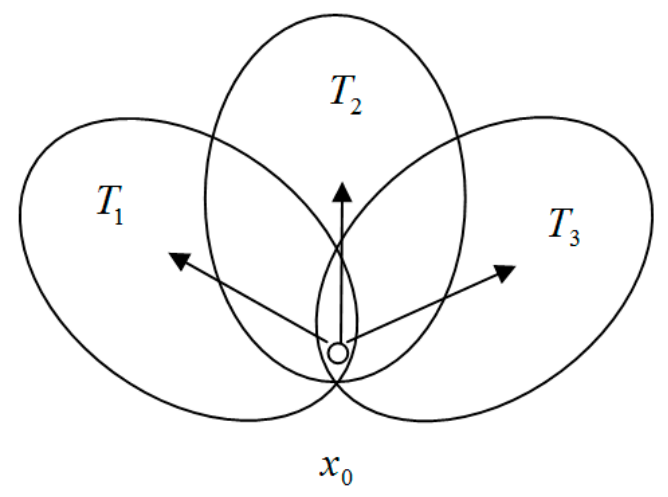

Таблица 1

\begin{tabular}{|c|c|c|}
\hline $\begin{array}{c}\text { Ёмкости поддеревьев } \\
\left(c\left(T_{1}\right) \geq c\left(T_{2}\right) \geq c\left(T_{3}\right)\right)\end{array}$ & $\begin{array}{c}\text { Применяемая оптимальная } \\
\text { стратегия }\end{array}$ & $\begin{array}{c}\text { Минимальное время } \\
\text { заполнения, } t_{\min }\end{array}$ \\
\hline$c\left(T_{1}\right)=c\left(T_{2}\right)=c\left(T_{3}\right)$ & Стратегия 1 & $\frac{V\left(T_{1}\right)+V\left(T_{2}\right)+V\left(T_{3}\right)}{2}$ \\
\hline$c\left(T_{1}\right)=c\left(T_{2}\right)>c\left(T_{3}\right)$ & Стратегия 2 & $\frac{V\left(T_{1}\right)+V\left(T_{2}\right)+V\left(T_{3}\right)}{2}$ \\
\hline$c\left(T_{1}\right) \geq c\left(T_{2}\right)+c\left(T_{3}\right)$ & Стратегия 3 & $\frac{V\left(T_{1}\right)}{2}$ \\
\hline$c\left(T_{1}\right)<c\left(T_{2}\right)+c\left(T_{3}\right)$ & Стратегия 4 & $\frac{V\left(T_{1}\right)+V\left(T_{2}\right)+V\left(T_{3}\right)}{2}$ \\
\hline
\end{tabular}

тимальная по времени стратегия заполнения, которая не является параллельной.

Обозначим через $n$ количество дуг, исходящих из корня.

Если $n \leq 3$, то возможны случаи, представленные на рис. 2.

\section{1. $n=1$}

Дерево состоит из одного поддерева $T_{1}$. Так как пропускная способность дуг равна 1 , то используемая мощность источника равна единице и минимальное время заполнения такого дерева равно сумме емкостей заполняемых вершин плюс количество дуг, то есть это время совпадает со временем заполнения в случае, когда мощность источника равна единице, [13].

Из сказанного следует, что время заполнения любого корневого поддерева $T_{i}$ равно $c\left(T_{i}\right)$ - ёмкости этого поддерева.

\section{2. $n=2$}

В случае с двумя поддеревьями, если $c(T)_{1}=c\left(T_{2}\right)=c(T)$, то параллельная стратегия существует и минимальное время заполнения $t_{\min }$ равно

$$
t_{\text {min }}=V(T)_{1}=V\left(T_{2}\right)=V(T) .
$$

Если $V(T)_{1} \neq V\left(T_{2}\right)$, то параллельной стратегии не существует и

$$
t_{\text {min }}=\max \left\{V(T)_{1} ; V\left(T_{2}\right)\right\},
$$

что меньше чем $V(T)_{1}+V\left(T_{2}\right)$ - время заполнения при мощности источника равной 1.

3. $n=3$

В случае с тремя поддеревьями возникает несколько возможных оптимальных стратегий их заполнения.

Эти стратегии подробно описаны в работе [14] и результаты можно свести в таблицу (табл. 1)

\section{Замечание 1.}

Обратим внимание на стратегию 3. Это единственный случай, когда мы не можем обеспечить параллельную, то есть максимально эффективную, заправку на протяжении всего времени заполнения графа. Однако стратегия является оптимальной среди возможных, так как в этом случае стратегии, обеспечивающей заполнение всех поддеревьев за меньшее вре- 


\section{В. В. Орлов}

мя, чем реализуемое этой стратегией, не существует.

При любом варианте заполнения дерева любое корневое поддерево заполняется из корня источником, мощность которого равна единице, поэтому время заполнения любого из поддеревьев численно равно его емкости. Таким образом, несмотря на то, что мы имеем дело с источником мощности два, его используемая мощность на любом поддереве равна 1 , следовательно, имеет место оценка

$$
t_{\text {зan. }} \geq \max _{i} V_{T_{i}}=V_{T_{1}}
$$

и поскольку время заполнения для стратегии 3 равно $V_{T_{1}}$, то эта стратегия оптимальна.

Перейдем к рассмотрению более общих случаев.

\section{Теорема 1.}

Пусть мощность источника равна 2, $n$ количество дуг, исходящих из корня, $n>3$. Будем считать, что поддеревья занумерованы таким образом, что $c\left(T_{1}\right) \geq c\left(T_{2}\right) \geq \ldots \geq c\left(T_{n}\right)$, тогда в случае

$$
c\left(T_{1}\right)>\sum_{i=2}^{n} c\left(T_{i}\right)
$$

время заполнения графа равно $V_{T_{1}}$; а в случае $c\left(T_{1}\right) \leq \sum_{i=2}^{n} c\left(T_{i}\right)$, существует параллельный процесс заполнения и время заполнения графа равно $t=\frac{\sum_{i=1}^{n} V\left(T_{i}\right)}{2}$.

\section{Доказательство.}

Случай 1:

$c\left(T_{1}\right)>c\left(T_{2}\right)+\ldots+c\left(T_{n}\right)$ аналогичен случаю для 3-х поддеревьев (строка 3 в таблице 1). Параллельная заправка здесь возможна только в течение части процесса заполнения. Оптимальная стратегия состоит в заполнении поддерева $T_{1}$ одновременно с $T_{2}$, после заполнения которого мы переключим освободившуюся единичную мощность на заполнение $T_{3}$ и т. д. К тому моменту, когда будет заполнено дерево $T_{n}$ в силу (2) дерево $T_{1}$ ещё не будет заполненным. В оставшееся время $V\left(T_{1}\right)-\left(V\left(T_{2}\right)+\ldots+V\left(T_{n}\right)\right)$ будет происходить дозаполнение поддерева $T_{1}$ и время всей заправки в этом случае составит $V\left(T_{1}\right)$. Меньше, чем $V\left(T_{1}\right)$ время заполнения графа быть не может (см. условие (1)). Поэто- му описанная стратегия оптимальна (но не параллельна).

Случай 2:

$c\left(T_{1}\right) \leq c\left(T_{1}\right)+\ldots+c\left(T_{n}\right)$ эта ситуация аналогична строкам в таблице для $n=3$ (строки 1 , $2,4)$, параллельная стратегия существует на протяжении всего процесса заполнения и её построение обеспечивается алгоритмом, описанным ниже. Этот алгоритм основан на двух моментах. Первое - необходимо уравнять ёмкости наибольших двух поддеревьев $T_{1}$ и $T_{2}$, второе - осуществлять последовательное уменьшение количества наименьших «хвостовых» поддеревьев $T_{n}, T_{n-1}, \ldots$ с помощью параллельной заправки. Тогда за конечное число шагов мы придём к ситуации заполнения либо двух равных ёмкостей (которые заполняются параллельно), либо трёх ёмкостей с двумя наибольшими равными, а это приводит к ситуации параллельной дозаправки, так как две наибольшие равны (строки 1, 2 в табл. 1).

Опишем алгоритм заполнения графа:

a) если $c\left(T_{1}\right)>c\left(T_{2}\right)$, то заправляем параллельно $T_{1}$ на величину $c\left(T_{1}\right)-c\left(T_{2}\right)$, и поддеревья, начиная с наименьшего, то есть $T_{n}, T_{n-1}$ и так далее. После этого может уменьшиться количество поддеревьев или уменьшится объём последнего оставшегося или одновременно уменьшится и количество «хвостовых» поддеревьев и объём последнего из оставшихся;

b) если $c\left(T_{1}\right)=c\left(T_{2}\right)$, то будем заполнять поддеревья попарно начиная с наименьшего. То есть сначала заполним поддеревья $T_{n}$ и $T_{n-1}$. Либо оба поддерева заполнятся одновременно и мы перейдём к следующей паре $T_{n-2}$ и $T_{n-3}$, либо заполнится только поддерево $T_{n}$, а объём поддерева $T_{n-1}$ только уменьшится и мы перейдём к заполнению пары $T_{n-1}$ и $T_{n-2}$;

c) когда количество поддеревьев станет $n \leq 3$ мы получим следующие ситуации:

1) $c\left(T_{1}\right)=c\left(T_{2}\right), \quad c\left(T_{3}\right)=0$, в этом случае просто дозаправляем параллельно первое и второе поддерево;

2) $c\left(T_{1}\right)=c\left(T_{2}\right)=c\left(T_{3}\right)>0$, этот случай описан в табл. 1 , строка 1 ;

3) $c\left(T_{1}\right)=c\left(T_{2}\right)>c\left(T_{3}\right)$, также см. табл. 1 , строка 2. 
Таким образом, нам удалось показать, что при мощности источника 2 и произвольном количестве поддеревьев графа (ёмкостей) в большинстве случаев можно организовать параллельный процесс заполнения так, чтобы мощность источника использовалась полностью на протяжении всего процесса заправки и время заполнения графа составит

$$
t=\frac{\sum_{i=1}^{n} V\left(T_{i}\right)}{2}
$$

Это невозможно в единственном случае если ёмкость первого поддерева больше суммы ёмкостей остальных, т. е. $c\left(T_{1}\right)>\sum_{i=2}^{n} c\left(T_{i}\right)$, при любых $n \geq 2$, для этого случая мы нашли единственную оптимальную стратегию, при которой время заполнения будет $V\left(T_{1}\right)$.

Пример 2.

Граф состоит из 5 поддеревьев ёмкостью: $12,5,4,3,2$. В этом случае $c\left(T_{1}\right) \leq c\left(T_{2}\right)+c\left(T_{3}\right)+$ $+c\left(T_{4}\right)+c\left(T_{5}\right)$ и $c\left(T_{1}\right)>c\left(T_{2}\right)$. По теореме 1 , в этом случае часть мощности источника подключаем к первому поддереву, а второй частью начинаем заполнять наименьшие поддеревья. Через семь шагов придём к ситуации: 5, 5,2 . Она соответствует стратегии 2 табл. 1, время заполнения будет равно 6, и всё время заполнения графа мы провели параллельно на всех шагах и это время оказалось равным 13.

$2^{0}$. Задача о заполнении растущего корневого дерева с вершинами положительной емкости одним источником мощности $m>2$

Рассмотрим сначала случай корневых поддеревьев равной ёмкости.

\section{Теорема 2.}

Пусть $m$ - мощность источника, $n$ - количество дуг, исходящих из корня, $n>m$ и ёмкости всех поддеревьев равны между собой, т. е. $c\left(T_{1}\right)=c\left(T_{2}\right)=\ldots=c\left(T_{n}\right)$. Тогда оптимальная стратегия, использующая параллельное заполнение графа, существует.

\section{Доказательство.}

По аналогии со случаем $m=2$, ясно, что если предложенная ниже стратегия параллельна, то она является оптимальной по времени заполнения. Если $n$ кратно $m$, то мы сможем последовательно полностью заполнять первые $m$ поддеревьев графа, затем пе- реходить к следующим $m$ поддеревьям и так далее до полного заполнения графа. Однако, если $n$ не кратно $m$, то на каком-то шаге применения этой стратегии количество оставшихся поддеревьев будет меньше, чем $m$, что не позволит нам полностью использовать мощность источника для заполнения оставшихся поддеревьев и мы потеряем время изза такого «простоя».

Покажем, что в случае, когда $n$ не кратно $m$ можно создать ситуацию «искусственной кратности» так, чтобы каждое поддерево заполнялось за $m$ равных промежутков времени.

Разделим ёмкость каждого поддерева на $m$. Будем заполнять параллельно по $m$ поддеревьев на величину $\frac{c\left(T_{i}\right)}{m}$, затем прерываться, и переключаться на следующие $m$ поддеревьев, и так далее ${ }^{1}$. Оставляя поддеревья частично заполненными, мы получаем возможность заново переподключаться к ним, когда нам это нужно. Всего таких переключений необходимо будет осуществить $m \cdot(n-1)$, если мы считаем уже подключенными первые $m$ поддеревьев. Таким образом, мы получили искусственно созданные $n \cdot m$ ёмкостей, которые можем записать в виде последовательности:

$$
\underbrace{123 \ldots n, 123 \ldots n, \ldots, 123 \ldots n}_{n \cdot m}
$$

Разрежем получившуюся последовательность на отрезки длины $m$ (см. рис. ниже)

$$
123, \ldots, m|m+1, \ldots,| n-m+1, \ldots, n .
$$

Каждый такой отрезок содержит $m$ разных натуральных чисел из промежутка $[1 ; n]_{N}$, поэтому поддеревья с такими номерами можно заполнять параллельно.

\section{Замечание 2.}

До сих пор мы не учитывали время, затрачиваемое на переключения от одного поддерева к другому. Если это время ненулевое, то общее время, затрачиваемое на эти переключения может существенно сказываться на времени заполнения. Алгоритм можно улуч-

${ }^{1}$ Заметим, что если ёмкость поддерева $c\left(T_{i}\right)$ не кратна $m$, то значения новых «искусственных» ёмкостей будут дробными. Это значит, что и время заполнения такой ёмкости будет также дробным. Будем считать, что дробное значение времени допустимо. 

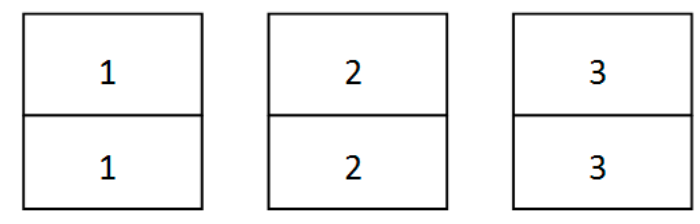
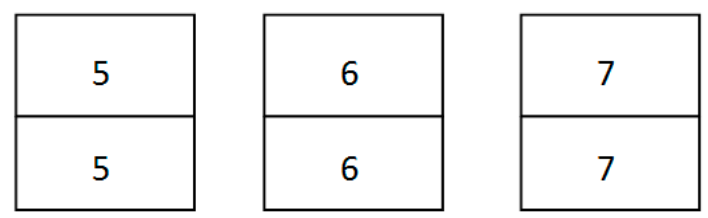

Puc. 3

шить, уменьшив количество переключений следующим образом. Пусть $n=m \cdot k+\delta$, $k \geq 2,1 \leq \delta \leq m-1$, где $\delta$ остаток от деления $n$ на $m$. Заполним сначала первые $m \cdot(k-1)$ деревьев параллельно, это возможно, так как количество деревьев кратно $m$. А затем к оставшимся $m+\delta$ деревьям применим процедуру «искусственная кратность». Это существенно уменьшает количество переключений.

\section{Пример 3.}

Рассмотрим ситуацию, когда имеется 7 равных по ёмкости поддеревьев и 1 источник мощности 2.

Если мы ёмкость каждого поддерева разделим на 2 равные части и пронумеруем эти части как показано на рис. 3, то последовательность номеров ёмкостей, и порядок их заполнения соответственно, будет иметь вид:

$$
12345671234567 .
$$

Можно использовать непосредственно алгоритм заполнения описанный в теореме 1 . Тогда количество переключений составит $(n-1) \cdot m=6 \cdot 2=12$.

Если же сначала полностью параллельно заполнить поддеревья 1 и 2, затем также 3 и 4, и уже затем применять общий алгоритм для поддеревьев 5, 6 и 7. В этом случае количество переключений составит 8 .

\section{Теорема 3.}

Пусть $m$ - мощность источника, $n$ - количество дуг, исходящих из корня, $n>m$, ёмкости $n-1$ поддерева равны между собой и ёмкость $n$-го поддерева меньше емкости каждого из них, т. е. $c(T)=c\left(T_{1}\right)=c\left(T_{2}\right)=\ldots$ $=c\left(T_{n-1}\right)>c\left(T_{n}\right)$. Тогда оптимальная стратегия, использующая параллельное заполнение гра-

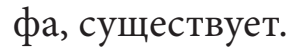

\section{Доказательство.}

Заполнение такого графа разбивается на два этапа. Сначала необходимо заполнить $n-1$ равные поддеревья на величину $c(T)-c\left(T_{n}\right)$. Это осуществляется алгоритмом описанным в теореме 1. Этим мы уравняли ёмкости всех поддеревьев до ёмкости $n$-го поддерева $T_{n}$. А теперь снова применим тот же алгоритм к $n$ поддеревьям уже ёмкости $c\left(T_{n}\right)$. Таким образом, мы полностью заполнили граф, используя максимальную мощность источника на протяжении всего времени заполнения. То есть нашли оптимальную стратегию, минимизирующую время заполнения графа.

\section{ЗАКЛЮЧЕНИЕ}

В настоящей работе нам удалось показать, что при мощности источника 2 и произвольном количестве корневых поддеревьев графа в большинстве случаев можно организовать параллельный процесс заполнения так, чтобы мощность источника использовалась полностью на протяжении всего процесса заправки. Это невозможно в единственном случае - если ёмкость первого поддерева больше суммы ёмкостей остальных поддеревьев. Но и для этого случая мы нашли оптимальную стратегию. Получены алгоритмы оптимального заполнения растущего из корня дерева потоком, создаваемым источником, находящемся в корне.

Найдены оптимальные стратегии заполнения для растущих из корня деревьев, имеющих $n>3$ корневых поддеревьев при мощности источника $m(n>m>2)$ для случая, когда ёмкости всех поддеревьев равны между собой и случая, когда ёмкости $n-1$ поддеревьев равны, а ёмкость $n$-го меньше остальных.

\section{СПИСОК ЛИТЕРАТУРЫ}

1. Форд, Л. Р. Потоки в сетях / Л. Р. Форд, Д. Р. Фалкерсон. - М. : Мир, 1966, 276 с.

2. Жилякова, Л. Ю. Теория ресурсных сетей: монография / Л. Ю. Жилякова, О. П. Кузнецов. - М. : РИОР: ИНФРА-М, 2017. - 283 с.

3. Жилякова, Л. Ю. Эргодические циклические ресурсные сети. I. Колебания и рав- 
новесные состояния при малых ресурсах / Л. Ю. Жилякова // Управление большими системами. Выпуск 43, М. : ИПУ РАН, 2013. C. 34-54.

4. Жилякова, Л. Ю. Эргодические циклические ресурсные сети. II. Большие ресурсы / Л. Ю. Жилякова // Управление большими системами. Выпуск 45, М. : ИПУ РАН, 2013. C. 6-29.

5. Kuznetsov, O. P. Nonsymmetric resource networks. The study of limit states. / O. P. Kuznetsov, L. Yu. Zhilyakova // Management and Production Engineering Review. Volume 2, Number 3, September 2011. - pp. 33-39

6. Жилякова, Л. Ю. Теория ресурсных сетей: монография / Л. Ю. Жилякова, О. П. Кузнецов. - М. : РИОР : ИНФРА-М, 2017. 283 с. - (Научная мысль). - URL: doi.org/ 10.12737/21451. - (Дата обращения 21.04.2019)

7. Ерусалимский, Я. М. Графы с вентильной достижимостью. Марковские процессы и потоки в сетях / Я. М. Ерусалимский, В. А. Скороходов // Известия вузов. Северо-Кавказский регион. Естественные науки. - 2003. № 2. - С. 3-5.

8. Ерусалимский Я. М. Графы с нестандартной достижимостью. Задачи, приложения: монография / Я. М. Ерусалимский, В. А. Скороходов, М. В. Кузьминова, А. Г. Петросян. Ростов-на-Дону : ЮФУ, 2009. - 195 с.

9. Ерусалимский Я. М. Случайные блуждания по графу-решётке и комбинаторные тождества / Я. М. Ерусалимский // Инженерный вестник Дона. - 2015. - № 2, Ч. 2 - 12 c. - URL: ivdon.ru/ru/magazine/archive/n2p2y2015/2964. - (Дата обращения 21.04.2019).

10. Erusalimskiy, I. On the Dynamic Flows in Networks / I. Erusalimskiy // Universal J. of Communications and Network. - 2014. - v 2. \# 6. - P. 101-105. - DOI: 10.13189

11. Ерусалимский, Я. М. Графы с затуханием на дугах и усилением в вершинах и маршрутизация в информационных сетях / Я. М. Ерусалимский, // Инженерный вестник Дона. - 2015. - № 1. - 12 c. - URL: ivdon.ru/ru/ magazine/archive/n1y2015/2782. - (Дата обращения 21.04.2019).
12. Жилякова, Л. Ю. Графовые динамические модели и их свойства / Л. Ю. Жилякова // Автоматика и телемеханика. - 2015. № 8. - C. 115-139.

13. Орлов, В. В. О заполнении вершин ориентированного графа / В. В. Орлов // Инженерный вестник Дона. - 2017. - Т. 47, № 4(47). - C. 114. - http://www.ivdon.ru/ru/ magazine/archive/n4y2017/4574. - (Дата обращения 21.04.2019).

14. Ерусалимский, Я. М. Методы оптимизации и инновации / Я. М. Ерусалимский, В. В. Орлов // Управление экономическими системами: электронный научный журнал. - 2018. - № 8 (114). - http://uecs.ru/ instrumentalnii-metody-ekonomiki/item/50572018-08-22-07-39-25. - (Дата обращения 21.04.2019).

15. Ерусалимский, Я. М. Потенциальный оператор, функция Грина на ориентированных графах и некоторые их приложения в квантовой механике / Я. М. Ерусалимский, Д. В. Степовой // Известия вузов. Северо-Кавказский регион. Естественные науки. - 2001, спецвыпуск. - С. 67-71.

16. Ерусалимский, Я. М. Дискретная математика: теория, задачи, приложения. - 3-е издание изд. - М. : Вузовская книга, 2000. $288 \mathrm{c}$.

17. Байрактаров, Б. Р. Алгоритм «веерной» оптимизации параметров разветвленных трубопроводных сетей / Б. Р. Байрактаров, В. Ч. Кудаев // Известия Высших учебных заведений. Северо-Кавказский регион. Естественные науки. - 2005. - № 2. - С. 2-5.

18. Байрактаров, Б. Р. Задача распределения потоков в кольцевых трубопроводных сетях / Б. Р. Байрактаров // Известия высших учебных заведений. Северо-Кавказский регион. Серия: Естественные науки. - 2005. № S1. - C. 1-9.

19. Кудаев, В. Ч. Задачи оптимального проектирования сетей Киргхофа: автореферат диссертации кандидата физико-математических наук: 05.13.16. - Ростов-на-Дону, 1993. - 18 c.

Орлов Владимир Владимирович - помощник генерального директора, Общество с ограниченной ответственностью «Гарантстрой, e-mail: diskret@mail.ru 


\title{
THE PROBLEM OF FILLING GROWING TREES WITH A SOURCE LOCATED AT THE ROOT
}

\author{
V. V. Orlov \\ LLC "Garantstroy"
}

\begin{abstract}
Annotation. The paper considers the optimization problem of filling growing trees with a source located at the root. Algorithms of work of this source are constructed for filling the graph that minimizes the time of filling the tree root. The case is completely considered when the number of arcs emanating from the root does not exceed 3 , and the source power does not exceed 2 . Then some more general cases are studied when the source power and the number of root subtrees are arbitrary. Theorems on the existence of optimal parallel tree filling strategies for some general cases are formulated and proved, and the optimal strategies themselves are described. It is proposed to improve the constructed tree filling algorithm by reducing the number of source power switching between root subtrees. Illustrative examples are provided for all results.

Keywords: optimization methods, efficient refueling, production planning, algorithms, root subtree, filling of vertices of an oriented graph.
\end{abstract}

Orlov Vladimir Vladimirovich - Assistant General Director, LLC “Garantstroy”, e-mail: diskret@ mail.ru 